\title{
Primary Cutaneous Marginal Zone Lymphoma
}

The Editor,

Sir,

A 64-year-old male was admitted to the hematology outpatient clinic with multiple irregular plaques with central necrosis ranging from 0.5 to 5 centimeters on medial surface of both legs. Lesions appeared gradually and progressed within 2 months (Figure). His history was unremarkable apart from fatigue. In past medical history, type 2 diabetes mellitus, hypertension, coronary artery disease, chronic obstructive pulmonary disease and hyperlipidemia was remarkable. He was using an angiotensin converting enzyme inhibitor and thiazide diuretic combination, a statin, $300 \mathrm{mg}$ acetylsalicylate daily, 2 grams of metformin and acarbose with meals. Physical examination revealed moderate pretibial edema apart from plaques. He had no hepatosplenomegaly or lymphadenopathy. Routine blood tests were normal. Peripheral blood smear was negative for atypical cells.

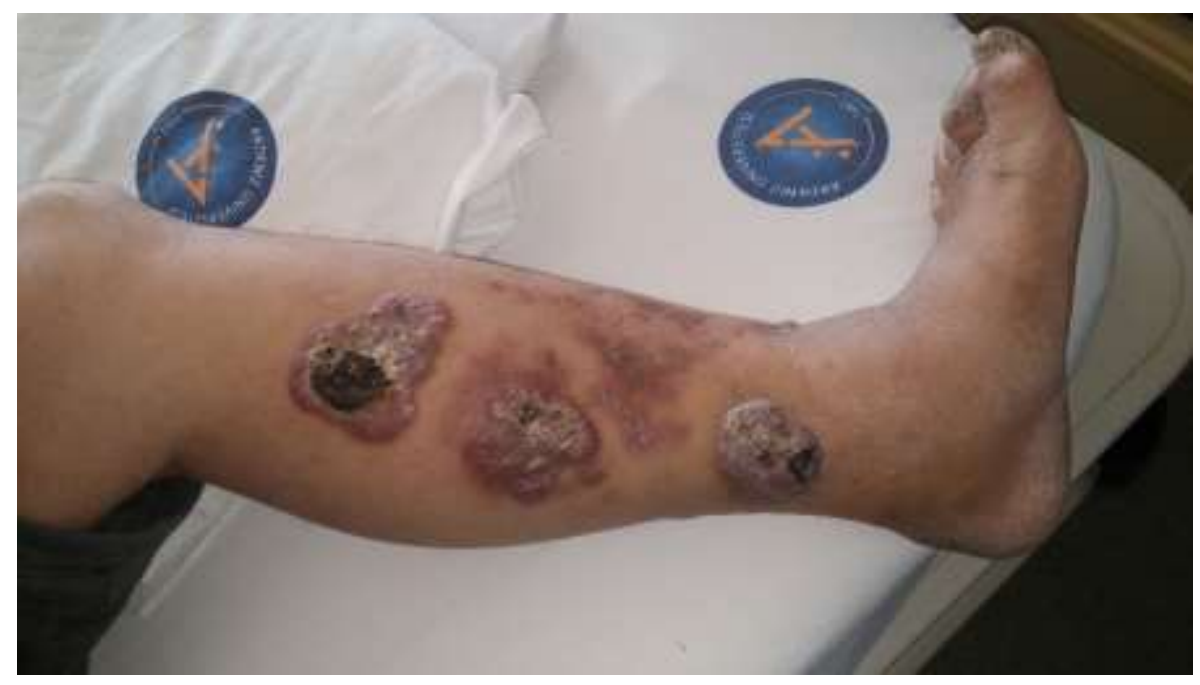

Figure: Patient's leg shows multiple irregular plaques with central necrosis ranging from 0.5 to 5 centimeters on the medial surface. 
A punch biopsy of the lesion showed CD20, CD79a, Pax-5 positive atypical B-cells producing kappa light chains; findings were consistent with marginal zone lymphoma. Ki-67 index was \%40. A full-body CT scan did not reveal any pathological lymph nodes or splenomegaly. Bone marrow biopsy was negative for lymphoma infiltration; bone marrow aspiration flow cytometric analysis, conventional cytogenetic and FISH analysis were negative as well. Both serum and urine immunofixation electrophoresis revealed no monoclonal bands. Patient was diagnosed as primary cutaneous marginal zone lymphoma and is being followed up for therapy.

Primary cutaneous B-cell lymphoma is a rare lymphoproliferative disorder manifested by cutaneous infiltration of monoclonal B cells without evidence of extracutaneous involvement. The etiology and pathogenesis of primary cutaneous B-cell lymphoma remain poorly understood. Herein, we aimed to report a rare case of primary cutaneous marginal zone lymphoma in an elderly male patient.

The term "primary cutaneous lymphoma" presents in the skin with no evidence of extracutaneous disease at the time of diagnosis. Among these various types of lymphomas, primary cutaneous marginal zone lymphoma is an indolent lymphoma composed of small B cells. They must be distinguished from systemic lymphomas, which may involve the skin secondarily and have a completely different clinical behavior and prognosis. Therapeutic mainstays for primary cutaneous marginal zone lymphoma have primarily included radiotherapy and surgery.

Keywords: Cutaneous lymphoma, marginal zone lymphoma, Turkey

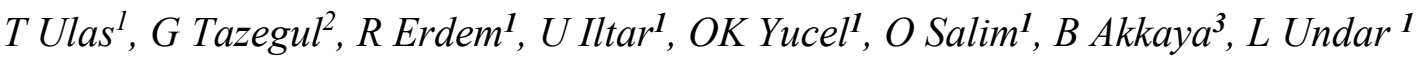

From: ${ }^{1}$ Division of Hematology, Department of Internal Medicine, ${ }^{2}$ Department of Internal Medicine, and ${ }^{3}$ Department of Pathology, Faculty of Medicine, Akdeniz University, Antalya, Turkey. 
Correspondence: Dr G Tazegul, Department of Internal Medicine, Faculty of Medicine,

Akdeniz University, Antalya,Turkey.E-mail:drgtazegul@gmail.com 


\section{REFERENCES}

1. Nicolay JP, Wobser M. Cutaneous B-cell lymphomas - pathogenesis, diagnostic workup, and therapy. J Dtsch Dermatol Ges 2016; 14: 1207-24.

2. Bahig H, Petrogiannis-Haliotis T, Pehr KL, Roberge D. Primary Cutaneous B-Cell Lymphoma in Young Monozygotic Twins: A Case Report. J Cutan Med Surg 2016; 20: $582-5$. 